\title{
Riesgos en la salud de agricultores por uso y manejo de plaguicidas, microcuenca "La Pila"
}

\author{
Health risks of farmers for using and handling pesticides in the watershed "La Pila"
}

\author{
Cristian Alexander Jiménez-Quintero' ${ }^{1}$, Andrés Pantoja-Estrada², Hugo Ferney Leonel ${ }^{3 *}$
}

\begin{abstract}
1 Estudiante Ingeniería Ambiental, Facultad de Ciencias Agrícolas, Universidad de Nariño. San Juan de Pasto, Colombia. e-mail: kajqui@hotmail.com

2 Estudiante Ingeniería Ambiental, Facultad de Ciencias Agrícolas, Universidad de Nariño. San Juan de Pasto, Colombia. e-mail: andespa001@gmail.com

3 Doctor en Ciencias Ambientales. Director y Docente del Departamento de Recursos Naturales y Sistemas Agroforestales. Facultad de Ciencias Agrícolas, Universidad de Nariño. San Juan de Pasto, Colombia. e-mail: hleonel2001@gmail.com
\end{abstract}

Jiménez-Quintero CA, Pantoja-Estrada A, Leonel HF. Riesgos en la salud de agricultores por uso y manejo de plaguicidas, microcuenca "la pila". Univ. Salud. 2016;18(3):417-431. DOI: http://dx.doi.org/10.22267/rus.161803.48

\begin{abstract}
Resumen
Introducción: El corregimiento de Cabrera del municipio de Pasto, fundamenta su economía en el sector agropecuario, actividad que demanda el uso y manejo de variados productos químicos cuyo empleo conlleva riesgos para la salud. Objetivo: Identificar los principales riesgos a los cuales están expuestos los campesinos agricultores de la microcuenca "La Pila", municipio de Pasto, por el uso y manejo inadecuado de plaguicidas en sus labores de producción. Materiales y métodos: Se comparó los resultados obtenidos en la simulación de la dispersión de plaguicidas a través del Software HYSPLIT libre y la evaluación de los riesgos por contacto inmediato identificados a través de criterios establecidos en la GTC45 de ICONTEC. Resultados: Se pudo determinar que el factor de riesgo al cual se encuentran sometidos los agricultores de la zona es de tipo químico; que la dispersión de dichos contaminantes alcanza una longitud máxima de dispersión de $250 \mathrm{~m}$ y que a pesar que los trabajadores conocen de la importancia de la implementación de Elementos de Protección Personal no los utilizan como barreras para atenuar e impedir la materialización de los peligros. Conclusiones: Los riesgos identificados son más latentes en los agricultores de la zona media y baja de la microcuenca.
\end{abstract}

Palabras clave: Factores de riesgo; mapa de riesgo; plaguicidas; impactos en la salud. (Fuente: DeCS, Bireme).

\begin{abstract}
Introduction: The village of Cabrera, in the municipality of Pasto, bases its economy on agriculture, which requires the use and handling of various chemicals that can cause health risks. Objective: To identify the main risks to which farmers are exposed for the use and improper handling of pesticides in their production work in the watershed called "La Pila" in the municipality of Pasto. Materials and Methods: The results obtained in the simulation of the dispersion of pesticides through HYSPLIT free Software and the risk assessment for immediate contact identified through established criteria in GTC45 ICONTEC were compared. Results: It was determined that the risk factor to which farmers in the area are subjected to is of chemical type; besides, the dispersion of these pollutants reaches a maximum length of dispersion of $250 \mathrm{~m}$ - However, despite the fact that workers are aware of the importance of the
\end{abstract}


implementation of Personal Protective Equipment, they do not use them as barriers to mitigate and prevent the realization of the dangers. Conclusions: The identified risks are latent in farmers of the middle and lower area of the watershed.

Keywords: Risk factors; risk map; pesticides; impacts on health. (Source: DeCS, Bireme).

\section{Introducción}

Díaz y Muñoz en 2013², definen los riesgos como aquellos elementos, eventos o acciones humanas que puede provocar daño en la salud de los trabajadores, en el sector rural uno de los riesgos por falta de medidas preventivas es el manejo y uso indiscriminado de plaguicidas. Según Bejarano 20112, los efectos negativos más comunes asumidos a estas sustancias, son dolores de cabeza, náuseas, vómitos, dolores de estómago y diarreas; sin embargo, la intensidad de estos efectos sobre la salud depende del tipo de plaguicida y su grado de toxicidad, cantidad o dosis de exposición, frecuencias de aplicación y utilización de medidas de protección personal ${ }^{3}$.

En la microcuenca "La Pila", localizada en el municipio de Pasto $(\mathrm{N})$, la economía depende de la producción agropecuaria, siendo su aporte de suma importancia al producto interno bruto del municipio de Pasto; ${ }^{4}$ sin embargo, para permanecer en el mercado, los agricultores han visto en la necesidad de aumentar la producción, con el uso de variados y agresivos productos químicos, entre ellos los plaguicidas; generando en el ámbito laboral peligros y riesgos para la salud por el uso inadecuado de éstos y la poca adopción de los elementos de protección para su manipulación.

A nivel internacional, García y Rodríguez en $2012^{5}$, presentaron una revisión sobre las principales causas y efectos en la salud de agricultores de Sinaloa, por el uso excesivo de plaguicidas. En el ámbito nacional, Tabares et al. en 20016, desarrollaron un análisis de los factores de riesgo por el uso y manejo de plaguicidas en las cuencas que alimentan a los sistemas de acueducto del departamento de Antioquia, determinando que el 53,9\% de los municipios encuestados presentaban el factor de riesgo, registrando 53 plaguicidas aplicados de todas las categorías toxicológicas. Por otro lado, Tabares y
López en 20117, determinaron que en Marinilla, Antioquia alrededor del $80 \%$ de los agricultores encuestados no utilizaban equipo de protección adecuado para realizar la labor; y la morbilidad más frecuente fue infección respiratoria aguda (78\%). López et al. ${ }^{8}$, reconoce la importancia de las prácticas de salud ocupacional en el ambiente de trabajo de los agricultores, así como la realización de estudios de diagnóstico de los contextos regionales bajo los cuales los campesinos manipulan dichas sustancias.

A nivel regional, Gómez y Ruíz en $2011^{9}$, en el municipio de Potosí (N); el 96,77\% de los agricultores encuestados, reconocían el peligro al que estaban expuestos al manipular plaguicidas; el 62,90\% no habían implementado los Elementos de Protección Personal (EPP) y sólo contaban con la protección disponible de acuerdo a sus recursos propios; para las labores de fumigación de cultivos de papa y cebolla se determinó riesgos de tipo químico con una valoración baja. En la microcuenca "La Pila", no se ha desarrollado ningún proceso de investigación pertinente respecto a riesgos en la salud por el uso de plaguicidas, lo que complejiza y dificulta la contextualización de escenarios posibles, factores de riesgo y naturaleza de los peligros; es por ello, que se observa la ineficiencia de los controles existentes, tanto en la fuente, medio e individuo.

La población de agricultores ha sido considerada especialmente vulnerable a ser afectada por riesgos asociados a plaguicidas, es así como Greenpeace International ${ }^{10}$, en un estudio sobre plaguicidas en el mundo, señala que los grupos de población particularmente expuestos o vulnerables por lo general son encabezados por agricultores que usan y manejan plaguicidas, demostrado a través del análisis de niveles de estas sustancias encontrados en la sangre y el cabello de dichos trabajadores. Por ende, el conocer los riesgos a los cuales se encuentran expuestos los trabajadores del sector agrícola de 
la microcuenca "La Pila", será beneficioso para que las instituciones y profesionales de la salud puedan proponer programas de prevención y promoción, que propenda por el bienestar integral de los agricultores.

La presente investigación, tuvo como objetivo, identificar los principales riesgos a los cuales están expuestos los agricultores de la microcuenca "La Pila", municipio de Pasto, por el uso y manejo inadecuado de plaguicidas en sus labores de producción. Para lo cual, se determinó cuáles son los plaguicidas más utilizados; su dispersión; y se analizó los posibles efectos inmediatos en la salud, asociados a la exposición por el uso de plaguicidas.

\section{Materiales y métodos}

La investigación se desarrolló en el segundo periodo del año 2015, en la microcuenca "La Pila", localizada al Noroeste de la capital del departamento de Nariño - Colombia, con una extensión de 1235 ha.; incluye las veredas: Buenavista, Cabrera Centro, La Paz, Duarte, Purgatorio, San José y Caracolito11, cuya organización se muestra en el mapa base de la zona, utilizado en los escenarios de análisis de las simulaciones realizadas para la determinación de la deriva de los plaguicidas utilizados (Figura 1). Se utilizó un marco metodológico descriptivo analítico, para lo cual, se empleó la metodología de Tabares y López ${ }^{7}$, correspondiente al número de agricultores entrevistados que comprende criterios adecuados para investigaciones que analizan la exposición a un contaminante y su relación con la salud de los posibles afectados ${ }^{12}$.

La población universo correspondió a 2650 habitantes ${ }^{11}$, distribuidos aproximadamente en 280 familias de las veredas ubicadas en la microcuenca "La Pila". Del total de las familias se seleccionó el 10\% (28), utilizando los siguientes criterios: a) predios que en el semestre B de 2015, el uso del suelo estaba destinado a cultivos de papa y cebolla; b) extensiones de cultivo mayores a una hectárea ( 1 ha) de acuerdo con Kraemer et al.13, Bonaparte ${ }^{14}$, quienes plantean que para extensiones mayores a 1 ha., el riesgo por exposición a plaguicidas aumenta; y c) agricultores que usaban agroquímicos. La unidad muestral fue el predio rural y la unidad de análisis los agricultores que usaban y manejaban plaguicidas.

La recolección de la información se realizó a través de fuentes primarias: encuesta dirigida, observación directa y registro fotográfico. Los datos se agruparon en las siguientes categorías de análisis: características de población, identificación de principales plaguicidas utilizados, formas de uso y manejo de los plaguicidas por parte de los cultivadores, factores de riesgo, efectos tempranos en la salud y medidas de prevención utilizadas. Los predios se seleccionaron en cantidad proporcional al número de cada una de las veredas. Para tal fin, con el apoyo del presidente de la junta de acción comunal de cada zona del corregimiento, se hizo visitas a las fincas seleccionándolas de acuerdo a los criterios antes mencionados.

\section{Consideraciones éticas}

La investigación protegió la privacidad del individuo sujeto de investigación; no se realizó ninguna intervención o modificación de las características físicas, biológicas o psicológicas de los sujetos de estudio; la participación de los individuos fue voluntaria y se solicitó el consentimiento informado, acorde con la Resolución 8430 de 1993, para la investigación en salud en Colombia7.

\section{Análisis de la Información}

La base de datos y el procesamiento de la información, se realizó en Excel 2016, mediante frecuencias, proporciones y análisis estadístico descriptivo simple. Con ayuda del Software Statgraphics Centuryón XVI, se realizó un análisis estadístico no paramétrico, determinando las variables que guardaban significancia estadística, mediante el cálculo del Chi cuadrado por método exacto. Lo anterior se complementó con un cruce multivariado, para establecer si existían asociaciones de población con variables agrupables y relaciones con pesos significativos, como respuesta a la metodología de estudios transversales descriptivo-analítico ${ }^{15}$. 


\section{Identificación de peligros y valoración de los riesgos}

La identificación de peligros y valoración de los riesgos en la salud de las personas que manipulan plaguicidas, con base a la metodología propuesta en la Guía Técnica Colombiana GTC 4516, cumpliendo con las siguientes actividades:

a) Definición del instrumento para recolectar información. Se optó por emplear la matriz de riesgos propuesta en la GTC 45.

b) Clasificación de los procesos, actividades y tareas. Se clasificó los procesos, las actividades y las tareas que comprenden la manipulación de plaguicidas, por parte de los productores campesinos del corregimiento de Cabrera, permitiendo preparar una lista de los procesos de trabajo y de cada una de las actividades que lo componen, con su respectiva clasificación, que incluye predios, instalaciones, personas $\mathrm{y}$ procedimientos.

\section{Identificación de los peligros}

a) Descripción y clasificación de los peligros. Con los agricultores, se estableció una lista de peligros, teniendo en cuenta el carácter de las actividades realizadas por ellos y los sitios en que se realiza dichas labores.

b) Efectos posibles. Se consideró consecuencias a corto plazo como los de seguridad en el uso adecuado, y las de largo plazo como las enfermedades asociadas a la exposición de plaguicidas.

c) Identificación de los controles existentes. Se relacionó la participación de los agricultores en la implementación de dichos controles, para reducir el riesgo relacionado a cada peligro generado en labores asociadas al uso de plaguicidas.

\section{Valoración del riesgo}

Se calificó el riesgo asociado a cada peligro, incluyendo dentro de esta, los controles existentes implementados; además, se tuvo en cuenta la eficacia de dichos controles, así como la probabilidad y las consecuencias si éstos fallan. Se definieron los criterios y se determinó la aceptabilidad del riesgo, para definir si el riesgo es aceptable o no.

\section{Evaluación de los riesgos}

La evaluación de los riesgos correspondió al proceso de determinar la probabilidad de que ocurran eventos específicos y la magnitud de sus consecuencias, mediante el uso sistemático de la información disponible.

Para evaluar el nivel de riesgo (NR), se usó la siguiente formula:

$$
N R=N P \times N C
$$

En donde: $N P=$ Nivel de probabilidad

$N C=$ Nivel de consecuencia

A su vez, se determinó el NP teniendo en cuenta:

$$
N P=N D \times N E
$$

En donde: $N D=$ Nivel de deficiencia

$N E=$ Nivel de exposición

\section{Simulación de la dispersión de plaguicidas}

Se utilizó el modelo de dispersión, Hybrid Single Particle Lagrangian Integrated Trajectory (HYSPLIT libre), desarrollado por el Air Resources Laboratory de la Administración Nacional Oceánica y Atmosférica (NOAA) de Estados Unidos; para ello, se tomaron los datos meteorológicos de REANALYSIS, que contiene datos desde el año 1948 al presente. Se seleccionó esta base de datos por ser la única que incluye los años que se deseaban abarcar para este trabajo.14

En la simulación se tomó sitio de aplicación cuatro predios en cada vereda de la microcuenca "La Pila", cumpliendo los parámetros de selección mencionados anteriormente. Los terrenos escogidos en cada vereda se consideraron como una unidad y dentro de esta área se trabajó con un punto fuente de la dispersión situado en el centro de la propiedad en estudio. El análisis se llevó a cabo por un periodo de 3 meses (periodo B 2015), valorando los riesgos, según la GTC4516. De acuerdo a la revisión bibliográfica, se estableció la 6 am como una hora de aplicación ${ }^{14}$.

Para la ejecución del modelo de simulación, se introdujeron en el programa, la cantidad de plaguicida utilizada en la fumigación, la cual varió en cada predio debido a las necesidades del cultivo; la distancia de dispersión (3-1 m); el 
tiempo en que tardo la fumigación (1 hora) y el formato de salida en el que se quería obtener el mapa de dispersión, por lo cual se utilizó imágenes de Google Earth, procesadas con ayuda del software ArcGis 10, obteniendo el mapa de dispersión de contaminantes, que sirvieron como herramienta fundamental al igual que la matriz de la GTC45, para el análisis de los riesgos por exposición a estos contaminantes.

\section{Resultados y discusión}

De la muestra poblacional estudiada se pudo determinar que el $80 \%$ fueron hombres y el $20 \%$ mujeres, en un rango de edad entre 40 y 50 años, de acuerdo al estudio no paramétrico realizado (Chi cuadrado exacto), ni la edad $(\alpha=0,05$ y $\mathrm{p}=0,21)$ ni el género $(\alpha=0,05$ y $p=0,11)$ presentaron relaciones significativas con respecto a la morbilidad por uso de plaguicidas, coincidiendo con el estudio multivariado aplicado a las encuestas, donde se demostró que no existen grupos que permitan establecer variables agrupables y que exhiban relaciones con pesos significativos de interés para el presente estudio.

En materia de morbilidad y exposición, todos los grupos están afectados de manera homogénea, esto se debe posiblemente a la sectorización primaria que se realizó para el muestreo, dado a que el escenario permitió el análisis sólo en agricultores que estuvieron en contacto directo e inmediato con los contaminantes. En contraposición Gómez y Ruiz ${ }^{9}$, encontraron diferencias estadísticas significativas entre la edad del agricultor y el tiempo de uso del plaguicida, cuyos resultados pudieron haberse presentado debido a que en dicho estudio no se hizo una sectorización previa de la población, determinando efectos en todos los pobladores.
En esta investigación al igual que la realizada por los anteriores autores se encontraron diferencias estadísticas entre el bajo nivel educativo y el conocimiento de los riesgos de los agroquímicos (Figura 1); tales diferencias se expresan por un porcentaje muy bajo (17\%) de las personas que utilizan plaguicidas y recibieron capacitación sobre uso y manejo de los mismos alguna vez en su vida, razón por la cual la mayoría de los encuestados (75\%) desconoce los posibles efectos que puede desencadenar la exposición a plaguicidas. Del mismo modo, la capacitación en pesticidas, la experiencia en el cultivo y la educación recibida por los agricultores, también estuvieron relacionadas de manera negativa con la intensidad en el uso de pesticidas, lo que explica que los agricultores con una buena formación y experiencia, son más propensos de conocer la información técnica necesaria para el uso eficiente de los plaguicidas; a su vez, es posible que sean conscientes de los efectos adversos de estas sustancias en la salud y el medio ambiente ${ }^{17}$.

Por otro lado, el estudio no paramétrico realizado, permitió evidenciar que pese a que no existen relaciones entre las variables agrupadas, estas pueden presentar relaciones particulares ${ }^{18}$ entre variables singulares que ameritan ser mencionadas, tales como, las veredas más afectadas que para el caso fueron Cabrera Centro y Buenavista; mostrando que la vereda de residencia influía significativamente en la presencia o ausencia de malestares después de la aplicación, bajo un Chi cuadrado de 8,01 en un cero de cero ${ }_{18}$ soportado en la dispersión de los contaminantes obtenida, que demostró como la dirección de la dispersión, afecta a algunas veredas más que a otras (Figura 1). 
Figura 1. Correlación entre veredas, capacitación y presentación de algún malestar*

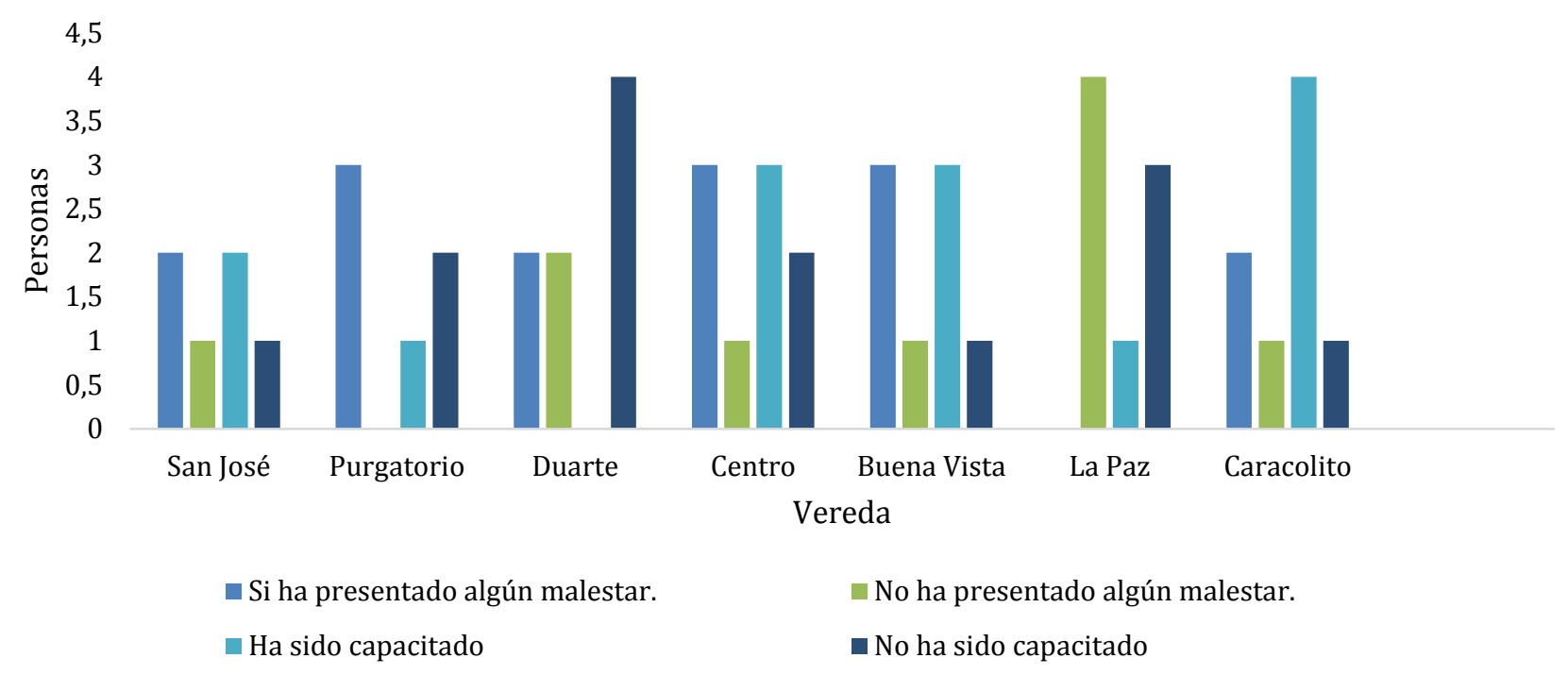

*Por chi cuadrado: fuerte relación para veredas y manifestar haber presentado algún malestar (índice de correlación 8,9); débil relación entre haber presentado un malestar y haber sido capacitado en el tema (índice de correlación 0,67).

No obstante, las capacitaciones recibidas han contribuido a que más del $90 \%$ manifiesten usar EPP, sin embargo, esto no es tan aplicable en la realidad, ya que tan solo se observó una persona usando EPP. Por su parte, las afecciones más destacables fueron mareos $(53 \%$ de los encuestados) y dolores de cabeza (61\%) (Figura 2 ), lo cual concuerda con resultados obtenidos en investigaciones realizadas por Sankoh et al. ${ }^{17}$, y en un estudio realizado por Tabares y López ${ }^{7}$, en donde la misma sintomatología la reportó el 85\% de la población encuestada; en la respuesta postaplicación los agricultores encuestados manifestaron presentar luego de finalizadas las actividades que implican el uso y manejo de plaguicidas, es aquella reportada por la literatura dentro de las características entorno a los efectos en la salud generados por sustancias como furadán, lorsban y cymoceb, los cuales son para el caso, los plaguicidas de mayor uso en las labores agrícolas de los campesinos de la microcuenca La Pila (Tabla 2).

En investigaciones realizadas se ha determinado que grupos poblacionales que viven cerca de cultivos, se encuentran expuestos a pesticidas que inciden en el deterioro del desarrollo neurológico de los niños principalmente ${ }^{18}$. La exposición a clorpirifos afecta la coordinación motora visual, la conducta y las habilidades para diferenciar la gama de colores, en el peor de los casos genera pérdida de memoria en los menores; se aúne a los resultados logrados, que la exposición al mancozeb y/o a su metabolito ETU van a influir directamente en el detrimento del aprendizaje verbal de la población infante.19

\section{Figura 2. Afecciones generales identificadas en los} agricultores encuestados

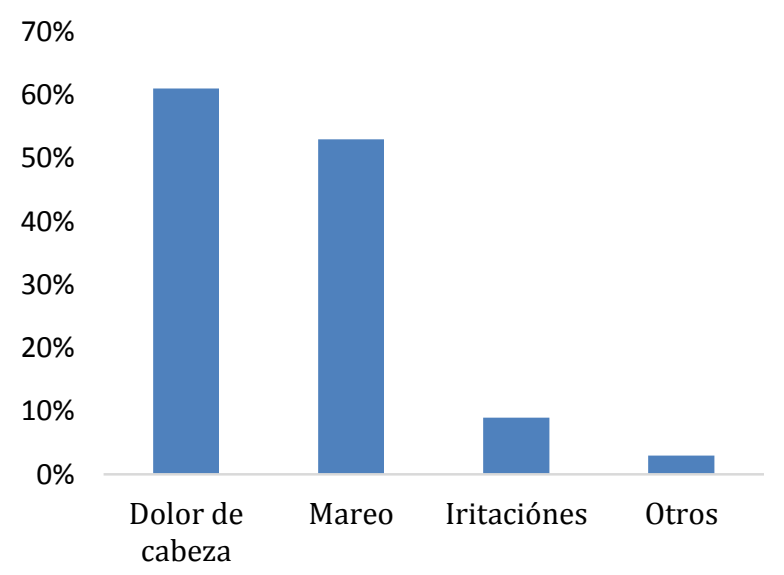

Otras variables que se tuvieron en cuenta en la morbilidad post aplicación (Tabla 1), fueron el hecho de si el individuo fumaba o comía durante 
la fumigación o cerca a esta; en periodos inmediatos o prolongados después de realizada la misma; mostrando una influencia débil con correlaciones entre 0.8 y 1.2 ; esto podría deberse a que en las áreas circundantes al cultivo se presentan concentraciones mayores a 0.001 $\mathrm{mg} / \mathrm{m}^{3}$ y según Abrutzky et al. ${ }^{20}$, en lugares donde generalmente no sobrepasan los niveles de contaminantes atmosféricos establecidos en la normatividad local, la contaminación produce serias afectaciones a la salud relacionadas con el aparato respiratorio y el sistema cardiovascular, y en casos extremos desemboca en la muerte
Además la extensión del cultivo determina un mayor uso de plaguicidas, dado a que según Sankoh et al., (2016) ${ }^{17}$ a mayor tamaño del predio de cultivo, mayor será el volumen de pesticida requerido; y este depende del poder adquisitivo de los agricultores y el área de la finca. Pese a que a no existe un protocolo estándar que sugiera un umbral prescrito para limitar la dosis a utilizar, esta práctica conlleva a un uso excesivo de pesticidas, alterando gravemente la calidad de salud de la población expuesta directamente y de aquellos que residen en cercanías al lugar de trabajo 21 .

Tabla 1. Factores que influyen en el análisis de la morbilidad post-aplicación

\begin{tabular}{|c|c|c|}
\hline Variable* & Categoría & $\begin{array}{c}\text { Total } \\
\mathrm{n}=\mathbf{2 8} \text { (valor porcentual\%) }\end{array}$ \\
\hline \multirow{7}{*}{$\begin{array}{l}\text { Destino de la ropa utilizada en la } \\
\text { fumigación }\end{array}$} & Se cambia antes de llegar a la casa (1) & $5(17,86)$ \\
\hline & La lava en el lugar de trabajo (2) & $1(3,57)$ \\
\hline & $\begin{array}{l}\text { La lava en la casa aparte de los demás miembros de } \\
\text { la familia (4) }\end{array}$ & $14(50)$ \\
\hline & $\begin{array}{l}\text { La deposita y lava junto con la de los otros } \\
\text { miembros de la demás familia (5) }\end{array}$ & $3(10,71)$ \\
\hline & Continúa con ella hasta la hora de acostarse (6) & $2(7,14)$ \\
\hline & Personas que respondieron (1) y (5) & $2(7,14)$ \\
\hline & Personas que respondieron (1) y (6) & $1(3,57)$ \\
\hline \multirow[t]{3}{*}{ Destino envases de plaguicidas } & Los quema (2) & $6(21,43)$ \\
\hline & Los guarda desocupados en la casa (6) & $6(21,43)$ \\
\hline & Personas que respondieron más de una opción & $16(57,14)$ \\
\hline \multirow[t]{2}{*}{ Fuma y manipula plaguicidas } & $\mathrm{Si}$ & $5(17,86)$ \\
\hline & No & $23(82,14)$ \\
\hline \multirow[t]{2}{*}{ Consume alimentos en el lugar de trabajo } & $\mathrm{Si}$ & $6(21,43)$ \\
\hline & No & $22(78,57)$ \\
\hline \multirow{4}{*}{$\begin{array}{l}\text { Se lava las manos antes de consumir } \\
\text { alimentos } \\
\text { Facilidad de aseo personal en el puesto } \\
\text { de trabajo }\end{array}$} & $\mathrm{Si}$ & $28(100)$ \\
\hline & No & \\
\hline & $\mathrm{Si}$ & $19(67,86)$ \\
\hline & No & $9(32,14)$ \\
\hline
\end{tabular}

Por otra parte, los agricultores de la microcuenca "La Pila", manifestaron que para el mejoramiento de la productividad de la papa y la cebolla es necesario el uso de los plaguicidas, evitando la pérdida de las cosechas por plagas y malezas. Se identificó que los agricultores que dependían únicamente del uso de pesticidas para la protección de sus cultivos mostraban usar mayor cantidad de estas sustancias que aquellos que no; a su vez, la frecuencia de aplicación de pesticidas varió de dos veces al mes hasta una vez a la semana, dependiendo del tipo de cultivo. La mayoría de los agricultores que usaban excesivamente los plaguicidas expresaban que estas sustancias son indispensables para obtener buenos rendimientos, determinando de esta manera la fuerte dependencia percibida entre plaguicidas y agricultores ${ }^{22}$. Tabares y López ${ }^{7}$, registraron que durante mucho tiempo se ha tenido la idea, entre los campesinos, de que la aplicación de químicos es la mejor manera para incrementar la producción, lo que se ha promulgado desde la oferta de las diferentes empresas fabricantes y proveedoras de plaguicidas, herbicidas $y$ otros productos químicos que circulan en el mercado agrícola. 
En la figura 3, se muestra el área de dispersión obtenida a partir de la aplicación de plaguicidas en los distintos predios estudiados. Se observa una región de dispersión dividida en cuatro frentes con un color característico para cada uno, en donde la dispersión se torna dominante tanto hacia el noreste como al suroeste del sitio de aplicación, concordando con la dirección dominante del recorrido del viento en la zona, determinada según mediciones realizadas en campo. Las zonas que determinan el nivel de riesgo más alto por exposición son las amarillas, sujetas a concentraciones mayores $0.001 \mathrm{mg} / \mathrm{ml}$, según el modelo HYSPLIT libre utilizado en la simulación. A partir del punto de aplicación los contaminantes al analizar alcanzan un rango de dispersión máxima de $2.5 \mathrm{Km}$ hacia la zona de Pasto, lo que genera problemas en la salud indirectos en poblaciones que no son agricultores, que incluso están fuera de los límites físicos de la microcuenca.

Las condiciones geográficas, principalmente la altitud hacia el norte, muestran detener el avance de la pluma y dirigirla hacia Buesaquillo, con un alcance de la dispersión máximo de tres kilómetros; ello demuestra que la aplicación inadecuada de plaguicidas logra trascender límites geográficos y naturales tal como lo afirma Rodricks ${ }^{23}$, haciendo de estos, un foco de contaminación difusa e intensa que requiere de atención y medidas de mitigación.

No obstante, un aspecto importante a destacar es la vida media de los ingredientes activos de los plaguicidas más utilizados en las labores realizadas por los agricultores de la zona, tales como carbofuran constituyente principal del furadán y de categoría altamente toxico; clorpirifos, componente base del lorsban; y mancozeb adicionado con cymoxanil, conformados estructurales del cymoceb, estos dos últimos de espectro no muy toxico (Tabla 2). Sin embargo, los plaguicidas más comúnmente usados por los campesinos de la microcuenca La Pila, corresponden a los mayormente empleados por los arroceros de Sierra Leona que incluyen chlopyrifos $(60 \%)$ y furadán $(20 \%)^{17}$; esto indica que los pesticidas usados en ambos contextos son un factor de peligro latente tanto para las personas expuestas a pesticidas como para el medio ambiente. Es así como, las sustancias analizadas en la investigación, no solo exhiben una dispersión muy difusa, sino también una permanencia en el ambiente prolongada, con tendencia a bioacumularse en los elementos de la microcuenca como lo menciona Burke ${ }^{24}$.

El área clasificada como de riesgo extremadamente peligroso, comprende aproximadamente a $500 \mathrm{~m}$ alrededor del predio de aplicación, por lo que se puede afirmar que toda edificación circundante, se encuentra en una zona de amenaza alta, frente a exposición a niveles de riesgo ${ }^{25}$. Con base en recorridos realizados, se pudo observar la tendencia de los habitantes a vivir junto al cultivo; esto quiere decir, que los tiempos post aplicación que permanecen los individuos en los predios tratados con plaguicidas, excede con creces las 24 horas recomendadas por la $\mathrm{FAO}^{26}$. Por lo tanto, la determinación de niveles "seguros" de exposición a plaguicidas individuales puede subestimar los efectos reales sobre la salud, ignorando también la exposición crónica a múltiples sustancias químicas constituyentes de variados productos, utilizados en las labores agrícolas realizadas por los campesinos en la consecución de los beneficios económicos necesarios para subsistir ${ }^{27}$.

Las actividades que están en contacto más inmediato con el producto post aplicación son las que tienen un nivel de riesgo más elevado o peligroso, como las que se realizan directamente cerca al cultivo ${ }^{23}$. Biofísicamente la comunidad se encuentra expuesta a niveles de riesgo altos; tanto por factores de dispersión, falta de concientización de los agricultores en la aplicación de buenas prácticas agrícolas (GAP) y manejo inadecuado de pesticidas, además del contexto de aplicación y las condiciones geológicas, hidrológicas y meteorológicas locales ${ }^{28}$; como por procesos inseguros que se realizan en las labores agrícolas evaluadas con nivel de riesgo I y II según la matriz GTC $45^{16}$. 
Figura 3. Riesgo por deriva de plaguicidas en la microcuenca La Pila (Simulación Hysplit)

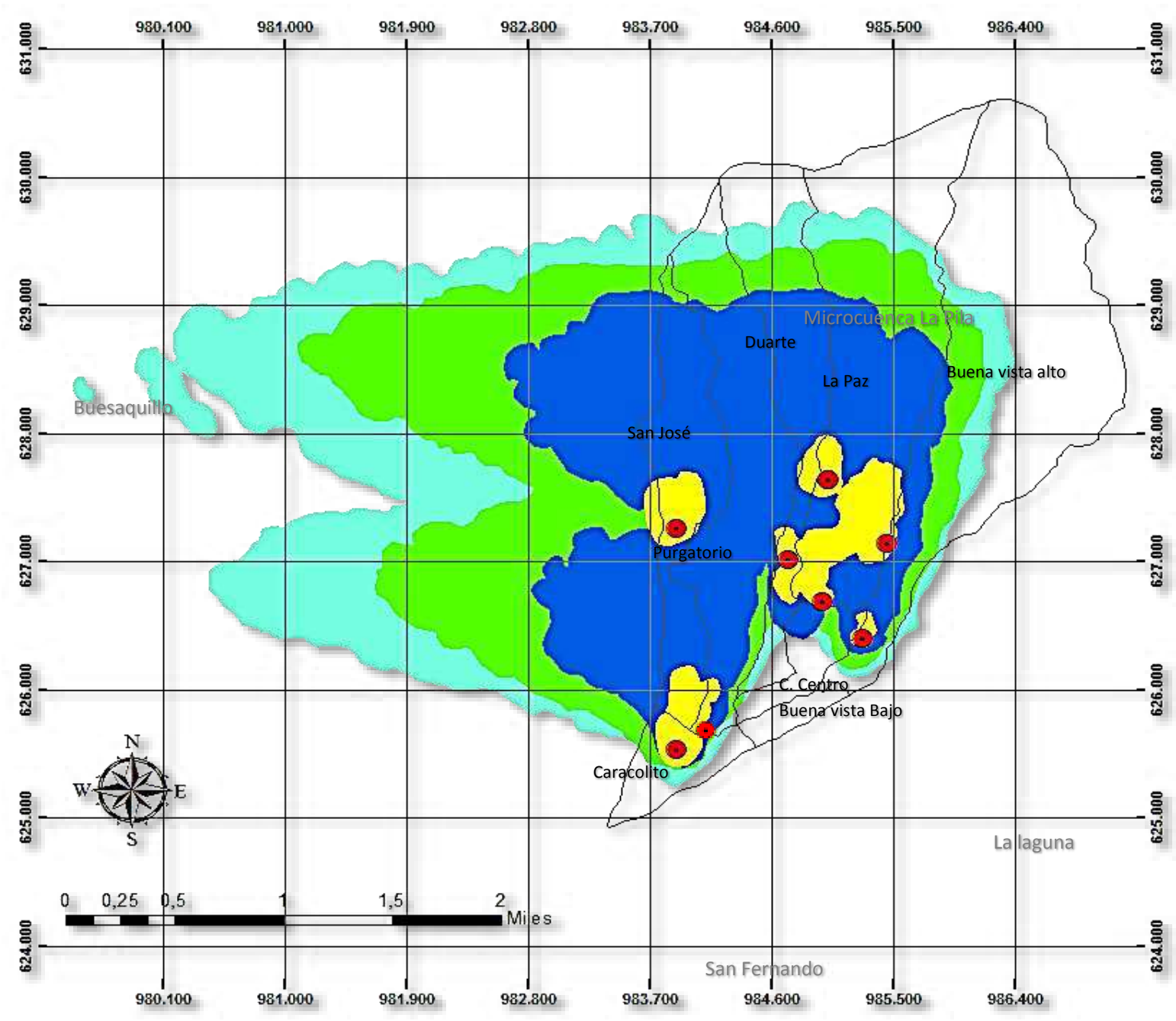

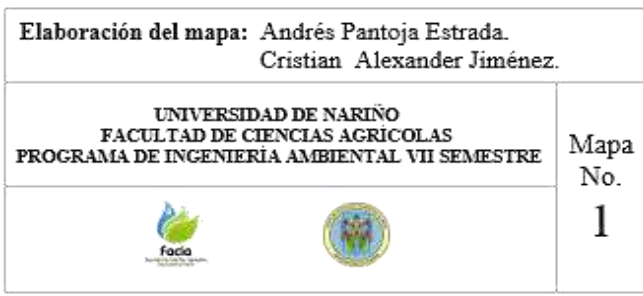

Escala 1:40.000

\section{Leyenda}

Riesgo extremadamente peligroso

R. altamente peligroso

R. medianamente peligroso

R. ligeramente peligroso

División política

Predios seleccionados

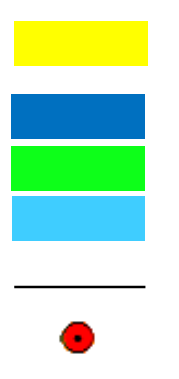


Algunas consideraciones que se pueden destacar de la matriz, fueron que tanto la clasificación como los efectos posibles, coinciden entre actividades, demostrando así, que pese a realizar distintos trabajos, la morbilidad inmediata post aplicación es igual indistintamente a factores socioeconómicos ${ }^{9}$, concordando con los datos estadísticos obtenidos. Sin embargo, aparentemente la situación puede empeorar debido a que los agricultores tienen la tendencia a aplicar plaguicidas similares a medida que producen cultivos análogos 28 , lo cual fue corroborado por medio de observación directa en la zona de estudio.

Dentro del proceso de recolección de información necesaria para el diligenciamiento de la matriz y el mapa de riesgo (Figura 3), y en contactos con líderes de las juntas de acción comunal de cada vereda, se identificaron avances en el mejoramiento de los procesos agrícolas orientados hacia la mejora de la calidad, para el acceso a mercados directos, generando así mayores beneficios económicos para los productores. En este marco dichas mejoras se miden en función de la aplicación de GAP, destacando entre ellas, el uso de EPP, y la aplicación de plaguicidas de categorías no muy tóxicas, que según la $\mathrm{FAO}$, son aquellos con ingredientes activos de categorías III y IV ${ }^{26}$.

Así mismo se identificaron los plaguicidas de uso más común en el sector (Figura 4) y su respectiva categoría toxicológica (Tabla 2), pudiendo establecer que el desconocimiento del nivel de peligrosidad de cada producto y las respectivas medidas de seguridad en su manipulación, conllevan a que el agricultor busque hacer más eficiente el control de plagas aplicando dosis mayores, sin considerar lo planteado por la FAO, a mayor categoría de peligro menor debe ser la dosis usada ${ }^{26}$. La aplicación de estos productos en la mayoría de los casos se realizaba de manera manual a través de bomba de espalda, y en muy pocos casos por medio de estacionarias.

Figura 4. Plaguicidas más utilizados por los agricultores entrevistados

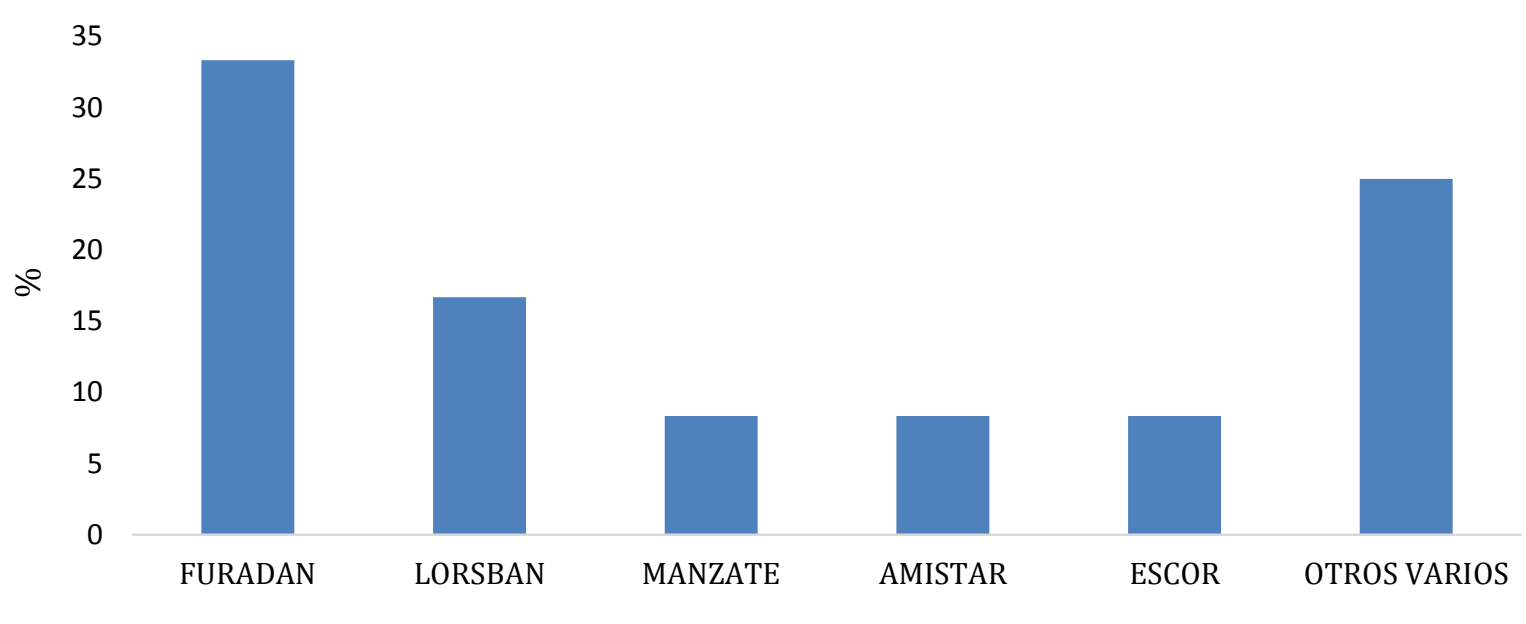

PLAGICIDA 
Universidad y Salud

Tabla 2. Plaguicidas usados por los habitantes de la microcuenca La Pila para el control de enfermedades agrícolas y sus efectos en la salud humana

\begin{tabular}{|c|c|c|c|c|c|}
\hline $\begin{array}{l}\text { Nombre } \\
\text { comercial }\end{array}$ & $\begin{array}{l}\text { Ingrediente } \\
\text { activo }\end{array}$ & $\begin{array}{l}\text { Categoría } \\
\text { toxicológica }\end{array}$ & $\begin{array}{c}\text { Objeto } \\
\text { de } \\
\text { control }\end{array}$ & $\begin{array}{c}\text { Grupo } \\
\text { químico }\end{array}$ & Efectos en la salud* \\
\hline
\end{tabular}

䓠

Irritación en la piel, asfixia, náuseas, vómitos, salivación, sudor frío, dolor abdominal, diarrea, lagrimeo, visión doble, miosis o contracción de la pupila, espasmos musculares, pérdida de coordinación y paro respiratorio.

Por poco tiempo (un día) a niveles bajos (miligramos) de clorpirifos puede causar mareos, fatiga, secreción nasal, lagrimeo, salivación, náusea, molestia intestinal, sudor y cambios en el ritmo cardíaco. La exposición oral de corta

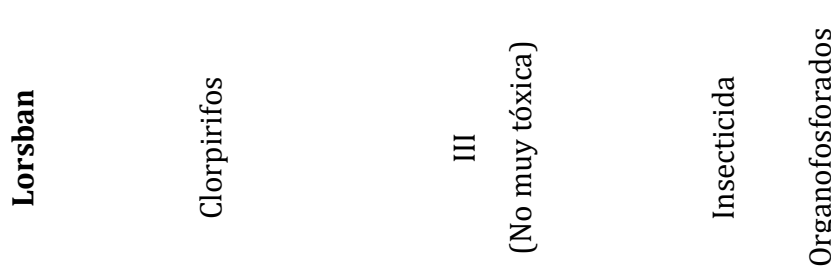
duración a niveles más altos (gramos) de clorpirifos puede causar parálisis, convulsiones, desmayos y muerte.

Otras consecuencias de la exposición al clorpirifos abarcan cambios de conducta o hábitos de sueños, cambios de humor y efectos en el sistema nervioso y en los músculos de las extremidades (que pueden manifestarse a través de sensaciones extrañas como insensibilidad $u$ hormigueo o como debilidad muscular).

A altos niveles ha causado defectos de nacimiento en animales de prueba, parálisis de las patas traseras y un aumento en la incidencia de atrofia retinal asociada con la vejez. Ha ocasionado tumores en la tiroides en animales de prueba, resultante de la formación de etilenotiourea (ETU).

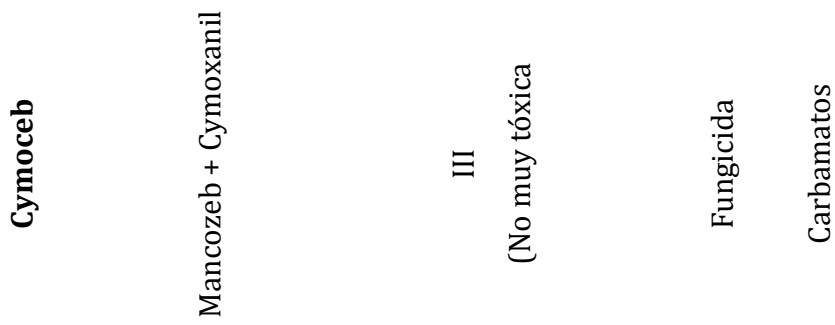
La ETU, una traza contaminante y sub- producto del metabolismo del mancozeb, primeramente afecta la tiroides. También ha causado otros efectos endocrinos, sanguíneos y en el hígado, tumores y defectos en el nacimiento en animales de experimentación. Los efectos tóxicos del mancozeb surgen de su metabolismo a pequeñas cantidades de ETU. El ETU inhibe la síntesis de la hormona tiroides, estimulando la secreción pituitaria de TSH provocando cambios tiroidales.

* La información correspondiente a la columna efectos en la salud, fue construida a partir de los aportes de: Binder et al. (2016) ${ }^{29}$; Instituto Nacional de Salud (2016) ${ }^{30}$; Pinilla et al. (2015) ${ }^{31}$; WHO et al. (2007) ${ }^{32}$; Dow AgroSciences Chile (2013) ${ }^{33}$; Nufarm Colombia (2016) ${ }^{34}$.

En la Tabla 3, se muestran los resultados obtenidos de la aplicación de la norma GTC-45. 
Tabla 3. Formato matriz GTC-45, identificación de peligros y valoración de riesgos

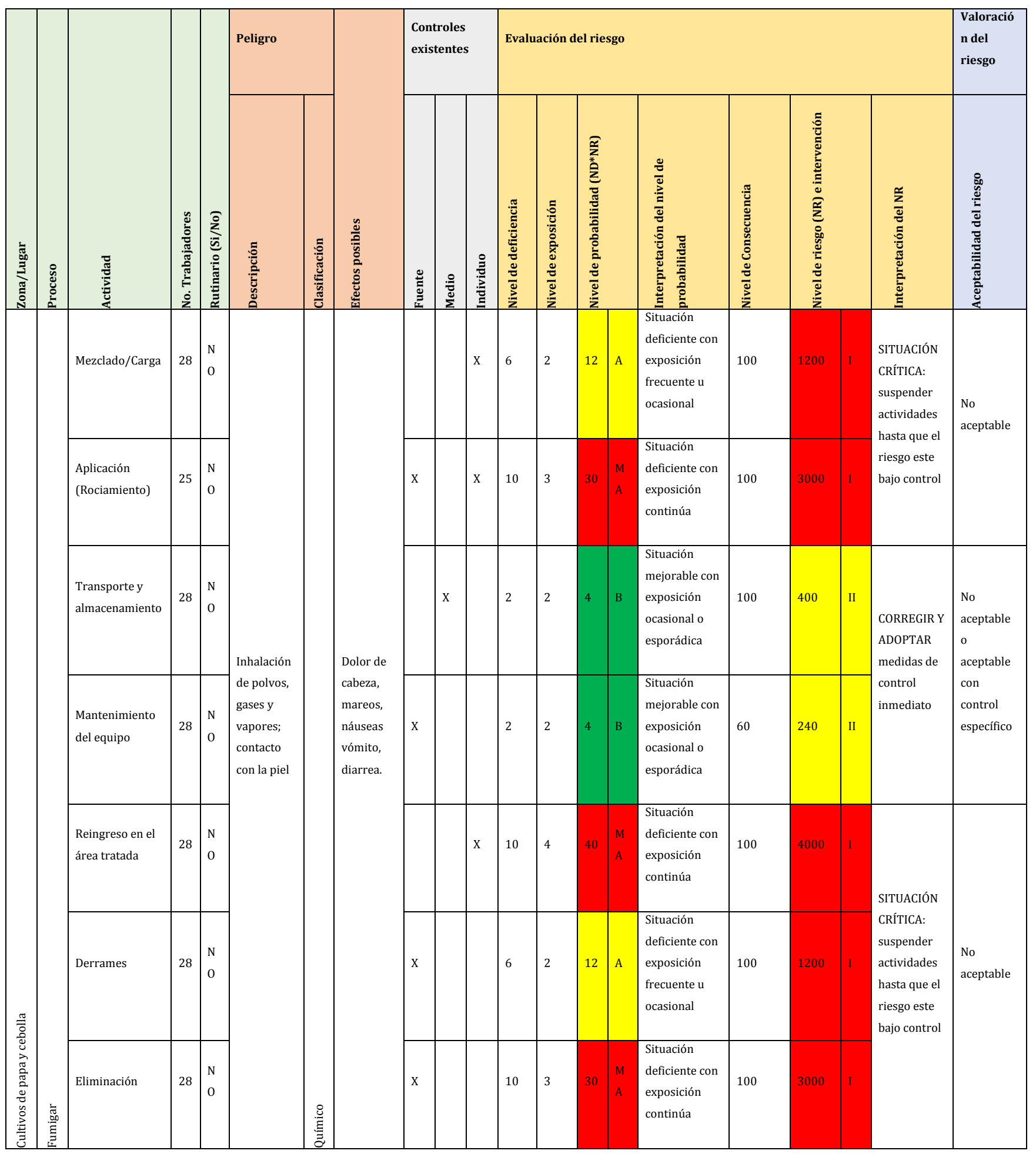


Las medidas de control que se propusieran en proyectos a futuro, deberán atender a la población de manera que garanticen mantener sus medios de vida, mientras se mitigan los aspectos generadores del peligro ${ }^{35}$, lo que difiere a la interpretación propuesta por la guía, ya que el enfoque industrial permite suspender actividades mientras se establecen las respectivas medidas de control; contrario al ámbito agrícola, donde los medios de subsistencia, no permitirían a los productores suspender la actividad mientras se realizan medidas de control convenientes. Por otro lado, las percepciones de los agricultores sobre el riesgo de los plaguicidas coincidieron en que el uso de plaguicidas plantea cierto riesgo para el medio ambiente $(100 \%)$ y la salud humana (100\%); a su vez, no se encontró ninguna asociación aparente entre las percepciones de los agricultores sobre el riesgo de los plaguicidas y la frecuencia en la aplicación de éstas sustancias en el control de plagas, resaltando la gran similitud con los resultados obtenidos por Jallow et al. ${ }^{22}$, en una investigación similar al caso analizado.

Se observó que a pesar de que existen regulaciones adecuadas, a la hora de evaluar la efectividad de estas en torno al control de los riesgos generados por el uso y manejo de plaguicidas, dichas medidas parecieran pasar por inexistentes, poniéndose de manifiesto deficiencias en su aplicación y recursos inadecuados, a la par de infraestructuras limitadas, que ponen a los trabajadores en alto riesgo de exposición a pesticidas. Por su parte, la disponibilidad inadecuada de equipos de protección; condiciones indebidas de aplicación y prácticas como, la aplicación rutinaria de plaguicidas altamente tóxicos por parte de trabajadores no capacitados, o largas horas de aplicación; instalaciones de almacenamiento y eliminación peligrosa; uso de equipos defectuosos y la mala calibración, y mantenimiento de los mismos; las instalaciones médicas y la falta de personal de salud capacitado, pueden elevar el riesgo para la salud de los trabajadores ${ }^{36}$.

\section{Conclusiones}

Se identificó que el único riesgo a la salud de los agricultores de la microcuenca está dado por el uso excesivo, permanente y manejo inadecuado de plaguicidas químicos, para el control fitosanitario de los cultivos de papa y cebolla, que habitualmente se usan tradicionalmente desde hace muchos años, como el Furadán, Lorsban y Cymoceb; siendo estos dos últimos, de una categoría toxicológica moderadamente peligrosa (III); para el caso del Furadán posee una toxicidad altamente peligrosa (IA), cuyo componente activo es el carbofuran, el cual ha sido sacado del mercado europeo hace muchos años por el alto riesgo que genera en la salud humana. El riesgo químico según la norma GTC-45 es no aceptable, lo que significa que este tipo de actividades deben ser suspendidas hasta que se controlen, lo que requiere de medidas de seguimiento y medias de contingencia.

Los síntomas que se suscitaron en las personas expuestas a niveles altos de plaguicidas, fueron dolor de cabeza y náuseas; seguido por las afecciones respiratorias y diarreas, que coinciden con la sintomatología temprana expuesta en información secundaria, que demuestran las personas que han estado sujetas a factores de riesgo por manipulación de plaguicidas.

Se pudo determinar que la exposición a riesgos por la dispersión de los contaminantes generados en la microcuenca "La Pila" afecta principalmente a los habitantes de la zona media y baja y en una pequeña proporción a los de la alta, además la pluma de dispersión también genera riesgos dentro de los habitantes que no viven dentro de la microcuenca, considerando que estos por la dirección y velocidad de los vientos son orientados hacia la ciudad de Pasto o hacia el sector conocido como Buesaquillo.

Conflicto de intereses: Ninguno declarado por los autores. 


\section{Referencias}

1. Díaz, O, Muñoz C. Aplicación de la GTC 34 y GTC 45 en una S.A.S. de servicios en HSEQ: estudio de caso. 2013: 4-6.

2. Bejarano J. Guía para la gestión ambiental responsable de los plaguicidas químicos de uso agrícola en Colombia. 2011: 22.

3. Machado A, Ruíz M, Sastre M, Butinof M, et al. Exposición a plaguicidas, cuidado de la salud y subjetividad. España y Portugal: Red de Revistas Científicas de América Latina y el Caribe; 2012.

4. Criollo C. Comunicación personal. 23 de noviembre de 2015.

5. García GC, Rodríguez MG. Problemática y riesgo ambiental por el uso de plaguicidas en Sinaloa. México: Red de Revistas Científicas de América Latina y el Caribe, España y Portugal; 2012: 1-12.

6. Tabares W, Galeano A, Bolívar J. Identificación de factores de riesgo por el uso y manejo de plaguicidas que abastecen los acueductos de las cabeceras municipales, Antioquia. 2001: 13-28.

7. Tabares J, López Y. Salud y riesgos ocupacionales por el manejo de plaguicidas en campesinos agricultores municipio de Marinilla. Antioquia: Red de Revistas Científicas de América Latina y el Caribe, España y Portugal; 2011.

8. López K, Pinedo C, Zambrano M. Prácticas de Salud Ocupacional y niveles de biomarcadores séricos en aplicadores de plaguicidas de cultivos de arroz en Natagaima-Tolima, Rev. Toxicol 32: 102-106p Colombia. 2015.

9. Gómez G, Ruíz E. Factores de riesgos ocupacionales a los cuales se encuentran expuestos los trabajadores informales de la agricultura en el municipio de Potosí, Nariño para el año 2010. 2011: 33-43.

10. Greenpeace International. Pesticides and our Health A Growing Concern, Research laboratories, Reino Unido. 2015.

11. Corponariño. Plan de Ordenamiento del Recurso Hídrico de la microcuenca La Pila, municipio de Pasto departamento de Nariño. 2009.

12. Kestenbaum B. Epidemiology and Biostatistics: An Introduction to Clinical Researchs. 29-31pp. Springer Science + Business Media. University of Washington, Seattle. 2009: 29-31.

13. Kraemer H, Yesavage J, Taylor J, Kupfer D. How can we learn about developmental processes from crosssectional studies, or can we?. Special articles. Crosssectional Studies AmjPsychiatry. Edition 157, 2000: 2.

14. Bonaparte E, Rubini M, Vera F, Barri F, Arguello C. Mapas de riesgo por deriva de plaguicidas en barrio Ituzaingó Anexo, Córdoba, Argentina. Universidad Nacional de Cordoba. 2012.

15. Siegel, Sidney. Non-parametric statistics for the behavioral sciences. McGraw-Hill. New York, US. 312pp. Edition XVII. 2005: 312.

16. ICONTEC. Guía Técnica Colombiana GTC-45. Guía para la identificación de los peligros y la valoración de los riesgos en seguridad y salud ocupacional. 2012.
17. Sankoh A, Whittle R, Semple K, Jones K, Sweetman A. An assessment of the impacts of pesticide use on the environment and health of rice farmers in Sierra Leone. Environment International xxx (2016) xxx-xxx.

18. Sprent P, Smeeton N. Applied nonparametric statistics methods, London, Library of Congress, third edition, 2001: 463.

19. Van Wendel De Joode B, Mora A, Lindh C, Hernández D, Córdoba L, Wesseling C, Hoppin J, Mergler D. Pesticide exposure and neurodevelopment in children aged 6-9 years from Talamanca, Costa Rica. Cortex XXX (2016) II4.

20. Abrutzky R.; Dawidowski L.; Murgida A. y Natenzon C. Contaminación del aire en la Ciudad Autonoma de Buenos Aires: El riesgo de hoy o el cambio climático futuro, una falsa opción. Ciência \& Saúde Coletiva, vol. 19, núm. 9, septiembre-, 2014: 3763-3773.

21. Deziel N, Beane L, Graubard B, Jones R, Hoppin J, Thomas K, et al. Relative Contributions of Agricultural Drift, Para-Occupational, and Residential Use Exposure Pathways to House Dust Pesticide Concentrations: Meta-Regression of Published Data. Environ Health Perspect DOI: 10.1289/EHP426. 2016.

22. Jallow M, Awadh D, Albaho M, Devi V, Thomas B. Pesticide risk behaviors and factors influencing pesticide use among farmers in Kuwait. Science of the Total Environment 574 (2017) 490-498.

23. Rodricks J. Calculated Risks: Understanding the Toxicity of Chemicals in Our Environment, Cambridge UK, Cambridge University Press; 2001.

24. Burke T. Applied epidemiology: theory to practice, Oxford, Oxford University press, Brownson; 2006.

25. Crissman C, Antle J, Cole D. Further evidence on pesticides, productivity and farmer health: Potato production in Ecuador, Canada, Department of Agricultural Economics And Economics, Montana State University; 1997.

26. FAO. Prácticas recomendadas para el manejo integrado del cultivo. Argentina. Red de Revistas Científicas de América Latina y el Caribe, España y Portugal. 2008.

27. Nicolopoulou-Stamati P, Maipas S, Kotampasi C, Stamatis P, Hens L. Chemical Pesticides and Human Health: The Urgent Need for a New Concept in Agriculture. Frontiers Public Health, Vol. 4, Article 148. 2016.

28. Mekonen S, Argaw R, Simanesew A, Houbraken M, Senaeve D, Ambelu A, Spanoghe P. Pesticide residues in drinking water and associated risk to consumers in Ethiopia. Chemosphere 162 (2016) 252-260.

29. Binder C, García G, Andreoli R, Diaz J, Feola G, Wittensöldner $M$, Yang J. Simulating human and environmental exposure from hand-held knapsack pesticide application: Be-WetSpa-Pest, an integrative, spatially explicit modeling approach. J. Agric. Food Chem., 2016, 64 (20): 3999-4008.

30. Instituto Nacional de Salud. Vigilancia y análisis del riesgo en salud pública. Protocolo de vigilancia en salud pública. Intoxicaciones por sustancias químicas. PROR02.006, versión 02 (2016): 1-75.

31. Pinilla G, Manrique E, Caballero A, Gómez E, Marín L, Portilla A, Sierra J, Prieto H, Oviedo D, Gamboa N. 
Neurotoxicología de plaguicidas prevalentes en la región Andina Colombiana. MÉD.UIS. 2015;27(3):57-67.

32. WHO, ILO, UNEP, IPCS, Ministerio de Trabajo y Asuntos Sociales de España, Instituto Nacional de Seguridad e Higiene en el Trabajo. Fichas internacionales de seguridad química: Carbofuran. España (2007): 1-3.

33. Dow Agrosciences Chile. Hoja de seguridad del Lorsban. Chile (2013): 1-7.

34. Nufarm Colombia. Tarjeta de emergencia del Cymoceb. Bogotá (2016): 1-4.
35. Crissman C. Environmental, and Health Tradeoffs in Agriculture: Pesticides, Washington, International potato center Economic, Kluwer Academic Publishers; 2001.

36. Yadav I, Devi N, Syed J, Cheng Z, Li J, Zhang G, Jones K. Current status of persistent organic pesticides residues in air, water, and soil, and their possible effect on neighboring countries: A comprehensive review of India. Science of the Total Environment. 2015; 511:123137. 